\title{
Autocrine/paracrine regulation of human endometrial stromal remodeling by laminin and type IV collagen
}

\author{
TETSUJI TANAKA, CHUNLIAN WANG and NAOHIKO UMESAKI \\ Department of Obstetrics and Gynecology, Wakayama Medical \\ University, Kimi-idera 811-1, Wakayama 641-0012, Japan
}

Received June 23, 2008; Accepted August 18, 2008

DOI: 10.3892/ijmm_00000059

\begin{abstract}
The biological roles of laminin and type IV collagen in human endometrial stromal tissues were investigated by the evaluation of the expression levels in human endometrial tissues using immunohistochemistry. In addition, normal human endometrial stromal cells were cultured in vitro on laminin- or type IV collagen-coated plates and subjected to cytological analyses. Cyclic production of laminin and type IV collagen were detected and the two productions were significantly increased in late proliferative and late secretory endometrial stromal cells. Unstimulated endometrial stromal cells proliferated with specific growth structures that varied depending on the extracellular matrix component coated on the culture plates. The expression levels of integrin subunits on endometrial stromal cells were sufficiently enhanced by $8 \mathrm{Br}-\mathrm{cAMP}$ treatment to mask any differences in the growth structures induced by the extracellular matrix components. 8Br-cAMP-stimulating stromal cells exhibited significant survival on laminin-coated plates, while 8Br-cAMP-deprived stromal cells, after 8Br-cAMP stimulation, showed significant survival on type IV collagen-coated plates. In conclusion, human endometrial stromal cells produce laminin and type IV collagen, and these productions are possibly regulated by ovarian estrogen and progesterone. Human endometrial stromal cells specifically bind to laminin and type IV collagen via integrins, and regulate endometrial stromal cell structures, viability and differentiation. Thus, laminin and type IV collagen may autoregulate human endometrial stromal remodeling during the menstrual cycle in an autocrine and paracrine fashion.
\end{abstract}

\section{Introduction}

Human endometrial tissues are remodeled each month. Immediately after menstruation, human endometrial cells

Correspondence to: Dr Tetsuji Tanaka, Department of Obstetrics and Gynecology, Wakayama Medical University, 811-1 Kimi-idera, Wakayama 641-0012, Japan

E-mail: obgywmu@wakayama-med.ac.jp

Key words: laminin, type IV collagen, fibronectin, endometrial stromal cell, apoptosis begin to proliferate under ovarian estrogen stimulation. After ovulation, the regenerated endometrial tissue differentiates under stimulation by ovarian progesterone in addition to estrogen and becomes able to receive embryo implantation. If the endometrium does not receive an embryo, the production levels of ovarian estrogen and progesterone decrease rapidly to induce endometrial shedding, i.e. menstruation. Therefore, remodeling of the functional human endometrium must involve the cyclic production of extracellular matrix (ECM) that occupies the intercellular spaces in addition to cell regeneration and proliferation. Laminin and type IV collagen (COL-4) are well-known major components of epithelial basement membranes in the human body, and have been reported to localize in the basement membrane structure of human endometrial epithelium $(1,2)$. Several studies have shown that these ECM components are produced at a high level in human deciduas $(3,4)$, while laminin was reported to inhibit endometrial stromal decidualization $(5,6)$. These ECM components were also reported to regulate trophoblast proliferation and differentiation during pregnancy (7-10). Furthermore, abnormal expression of these ECM components in the endometrium was reported in a patient, infertile from an unknown cause (1).

The cyclic changes in the expression levels of laminin and COL-4 during the menstrual cycle have not yet been clarified, although these components were reported to localize in decidualized tissues (3-4). Cyclic remodeling of the human endometrium must be regulated by cyclic proliferation, differentiation, morphological changes and cell death during the menstrual cycle. However, the effects of laminin and COL-4 on these cyclic functional and morphological changes in the endometrium have not been thoroughly investigated. Therefore, in the present study, we investigated the production levels of laminin and COL-4 in endometrial stromal cells (ESCs) as well as the effects of these components on ESC viability and the structures of ESC colonies.

\section{Materials and methods}

Immunohistochemistry. After receiving written informed consent from participants, normal human endometrial tissues were obtained from 22 patients (aged 25-49 years) with normal menstrual cycles whose uteri were surgically removed due to cervical cancer or leiomyoma and 4 postmenopausal women (aged 51-55 years) with cervical cancer. 
The tissue samples were fixed in $10 \%$ formalin solution and embedded in paraffin. Serial sections, $3 \mu \mathrm{m}$ thick, were cut and mounted on gelatin-coated glass slides. To confirm the endometrial dating, each sample was stained with H\&E. Tissues were classified into the following five menstrual stages according to the menstrual cycle from the patients' chart records and histopathological findings: mid-proliferative period (MP, within 9 days after initiation of menstruation, $\mathrm{n}=5$ ); late proliferative period (LP; from 10 days after initiation of menstruation to ovulation, $\mathrm{n}=6$ ); mid-secretory period [MS, postovulatory period with some decidualization $(\leq 5 \%)$, $\mathrm{n}=5$ ]; late secretory period (LS, endometrial tissues with a large amount of decidualization, $n=6$ ) and post-menopausal period (PM, at least 2 years after spontaneous menopause, $\mathrm{n}=4$ ).

Immunohistochemical examinations were carried out using an avidin-biotin peroxidase complex (ABC) technique. Briefly, deparaffinized sections were washed three times with phosphate-buffered saline (PBS) for $5 \mathrm{~min}$ at room temperature. After microwaving, the sections were treated with $0.3 \%$ hydrogen peroxide in absolute methanol to block endogenous peroxidase activity, and washed five times with PBS at room temperature. After blocking with normal horse serum (Dako Carpinteria, CA) (1:20 dilution) for collagen IV staining or normal swine serum (Dako) (1:20 dilution) for laminin staining, the sections were incubated with mouse anti-human type IV collagen IgG (Dako) (1:300 dilution) or rabbit anti-human laminin IgG (Dako) (1:600 dilution) overnight at $4^{\circ} \mathrm{C}$, and then washed five times with PBS. Then, the sections were incubated with biotinylated horse anti-mouse IgG (Dako) (for collagen IV staining) or biotinylated swine anti-rabbit IgG (Dako) (for laminin staining) for $30 \mathrm{~min}$ at room temperature, followed by incubation with avidin-biotinylated horseradish peroxidase complexes (Vector Laboratories Inc., Peterborough, UK) for $60 \mathrm{~min}$ at room temperature. After five washes with $0.05 \mathrm{M}$ Tris-buffered saline, $\mathrm{pH} 7.4$, positive reactions were visualized with $0.03 \%$ hydrogen peroxide and $0.1 \% 3,3$ diaminobenzidine-tetrahydrochloride (DAB) (Dako) in Tris buffer. After another five washes, the sections were counterstained with Mayer's hematoxylin (Nakalai, Kyoto, Japan). In order to test the specificity of the immunolabeling, negative control sections from each sample were stained by the same protocol without the first antibody. For analysis, 400 stromal cells were counted in each section and the immunoreactivity ratios (\%) were calculated. Graphical data were plotted as a mean \pm SD and data comparisons were analyzed using t-tests $(n=4-6)$. Values of $p \leq 0.05$ were considered to indicate statistically significant differences.

Culture of ESCs. We used normal human ESCs obtained from patients with non-endometriotic leiomyoma and normal menstrual cycles to study the effects of ECM components on stromal cell culture. The patients provided written informed consent for the collection of normal endometrial cells from their uteri after surgical excision. The endometrial tissue samples were cut into small fragments with scissors and incubated in medium containing $0.1 \%(\mathrm{w} / \mathrm{v})$ collagenase (Wako Chemicals, Tokyo, Japan) for $30 \mathrm{~min}$ at $37^{\circ} \mathrm{C}$. After the removal of epithelial cells by filtration through a $40-\mu \mathrm{m}$ nylon mesh, the remaining cells were cultured. Non-adherent cells were removed by several extensive washes. Finally, $\geq 98 \%$ of the residual adherent cells were found to be positive for vimentin, a stromal cell lineage-specific marker (data not shown). These cells were used for subsequent experiments. ESCs were cultured in OPTI-MEM (Gibco BRL, Gaithersburg, MD), 5\% fetal calf serum (FCS, Equitech Bio Inc., Ingram, TX), penicillin (100 U/ml, Gibco BRL), streptomycin (100 $\mu \mathrm{g} / \mathrm{ml}$, Gibco BRL) and 17ß-estradiol (100 nM, Sigma Chemical Co., St. Louis, MO).

Cell viability assay. Viable cell numbers were assayed using a cell proliferation assay kit (XTT; Boehringer Mannheim, Mannheim, Germany). Briefly, ESCs were detached by $0.25 \%$ trypsin/ $1 \mathrm{mM}$ EDTA (Gibco BRL) and cultured in the previously described estradiol-containing medium in 96-well culture plates (2500 cells/well). On days $1-14,0.5 \mathrm{mM} 8 \mathrm{Br}-$ cAMP (Sigma Chemical Co.) was added to the medium, and the culture medium was exchanged every 3 days. On day 14 , the numbers of viable $8 \mathrm{Br}$-cAMP-stimulating cells were assayed using the XTT kit. To examine the effects of $8 \mathrm{Br}-$ cAMP deprivation on cell viability, ESCs were cultured with $8 \mathrm{Br}$-cAMP until day 10 , washed with media without $8 \mathrm{Br}-$ cAMP on day 11 and cultured in $8 \mathrm{Br}$-cAMP-free media for 3 days, before the numbers of viable cells were assayed using the XTT kit..

The effects of ECM components on the numbers of viable cells were examined by culturing ESCs on type I collagen-, type IV collagen-, fibronectin- or laminin-coated 96-well plates (all from Becton Dickinson, Franklin Lakes, NJ). Noncoated 96-well plates purchased from Nalge Nunc International (Copenhagen, Denmark) were used for negative control cultures.

All experiments were performed in triplicate. Graphical data were plotted as a mean \pm SD and data comparisons were analyzed using t-tests $(n=12)$ and/or one-way ANOVA. Values of $\mathrm{p} \leq 0.05$ were considered to indicate statistically significant differences.

Semiquantitative flow cytometry. ESCs were detached from tissue culture dishes using $3 \mathrm{mM}$ EDTA in PBS, and stained according to the following procedure. Cells $\left(3 \times 10^{5}\right)$ were incubated with an excess of one of the primary antibodies for $20 \mathrm{~min}$ at $4^{\circ} \mathrm{C}$, and then washed twice with washing buffer (PBS containing 2\% FCS and $0.1 \% \mathrm{NaN}_{3}$ ). Then, the cells were reacted with FITC-conjugated goat anti-mouse $\mathrm{IgG}$ $(\mathrm{H}+\mathrm{L})\left(\right.$ Dako) as a secondary antibody for $20 \mathrm{~min}$ at $4^{\circ} \mathrm{C}$, and washed twice. Finally, the cells were suspended in $200 \mu 1$ of washing buffer and analyzed using a FACScalibur ${ }^{\mathrm{TM}}$ (Becton Dickinson). The primary antibodies used were: mouse antihuman CD49a monoclonal antibody (clone TS2/7, Serotec Ltd., Oxford, UK); mouse anti-human CD49b monoclonal antibody (clone 31H4, Serotec Ltd.); mouse anti-human CD49c monoclonal antibody (clone 11G5, Cymbus Biotech Ltd., Chandlers Ford, UK); mouse anti-human CD49d monoclonal antibody (clone 44H6, Cymbus Biotech Ltd.); mouse anti-human CD49e monoclonal antibody (clone SAM1, Beckman Coulter Japan, Tokyo, Japan); and mouse anti-human CD49f monoclonal antibody (clone 4F10, Cymbus Biotech Ltd.). ESCs treated with the secondary antibody alone were used as negative controls. 


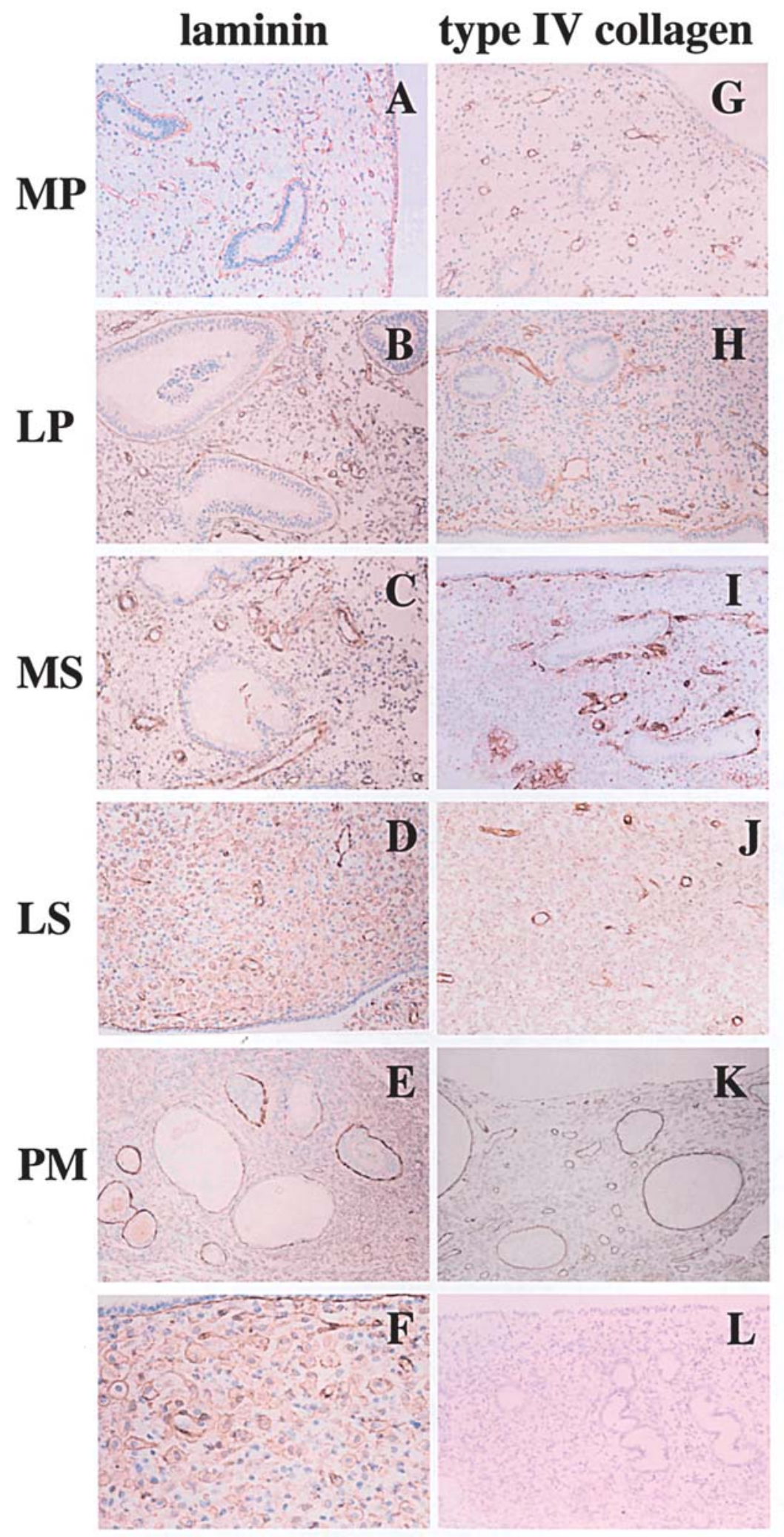

Figure 1. Expression profiles of laminin and type IV collagen in human endometrial stromal tissues. The endometrial tissues were classified into the following groups: MP, mid-proliferative period; LP, late proliferative period; MS, mid-secretory period; LS, late secretory period; and PM, postmenopausal endometrium. (A-F) Laminin expression profiles. (G-K) Type IV collagen expression profiles. (L) Negative control. (F) Magnified decidualized stromal tissue.

\section{Results}

Production of laminin and COL-4 by normal human ESCS. Normal human endometrial tissues were analyzed for their production of laminin and COL-4 by immunohistochemistry.
Strong immunoreactivities for laminin and COL-4 were found in basement membrane-like structures in the epithelial layers. ESCs at the LP period were weakly stained while decidualized stromal cells showed strong immunoreactivity (Fig. 1). The ratios of the laminin-positive stromal cells 
$\mathbf{A}^{\prime}$

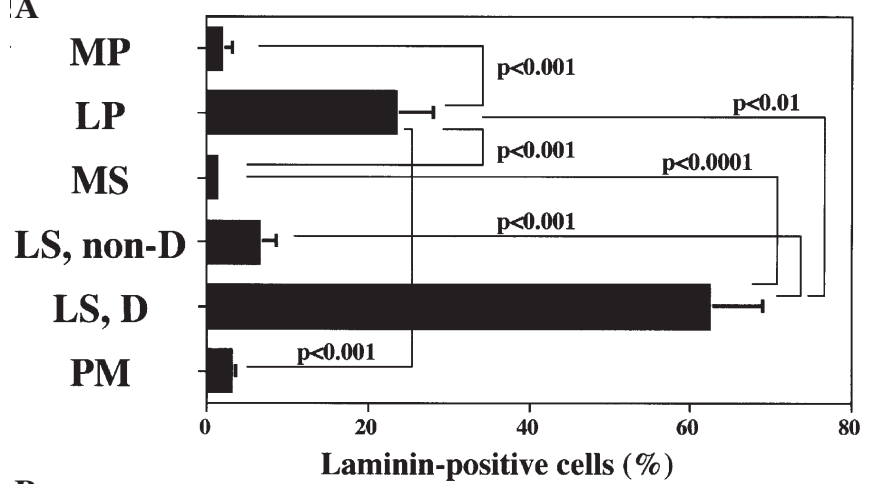

B

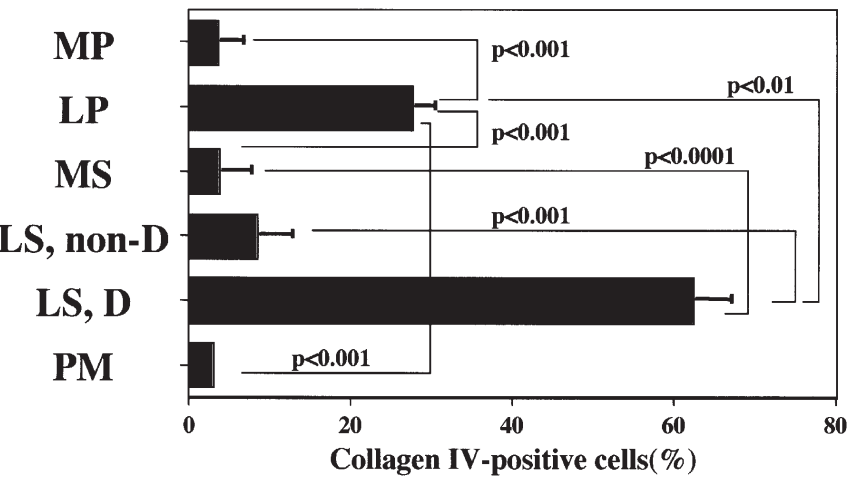

Figure 2. Expression levels of laminin and type IV collagen in human ESCs. The numbers of laminin-positive and COL-4-positive ESCs were counted and the positive ratios (\%) were compared. The endometrial tissues were classified into the following five groups: MP, mid-proliferative period; LP, late proliferative period; MS, mid-secretory period; LS, late secretory period; and PM, postmenopausal endometrium. Among the LS endometrial tissues only, the numbers of immunoreactive stromal cells for laminin and COL-4 were individually counted among the non-decidualized stromal cells (LS, non-D) and decidualized stromal cells (LS, D).

and COL-4-positive stromal cells were significantly increased in the LP and LS periods (Fig. 2).

Expression of integrins on normal human ESCs. Integrins are receptors for ECM components. Flow cytometric analyses were performed to examine whether integrin subunits were expressed on unstimulated normal human ESCs and $8 \mathrm{Br}$ cAMP-stimulated ESCs (Fig.3). Unstimulated ESCs exhibited weak expression levels of CD49a and CD49e on their cell surface. After stimulation with $8 \mathrm{Br}-\mathrm{cAMP}$, a strong decidualization-inducing reagent, ESCs exhibited increased levels of integrin subunit expression on their cell surface, with the exception of CD49d. Markedly higher expression levels of CD49a, CD49c CD49e and CD49f were found on 8Br-cAMP-stimulated ESCs.

Morphological differences of ESCs cultured on ECM component-coated plates. If the integrins on human ESCs can bind to specific ECM components, morphological differences of cultured ESCs should be observed depending on their interactions with individual ECM components. To investigate this hypothesis, the microscopic findings for ESCs cultured on various ECM component-coated dishes were compared. In the absence of $8 \mathrm{Br}$-cAMP, clear differences in the structures of ESC colonies growing on

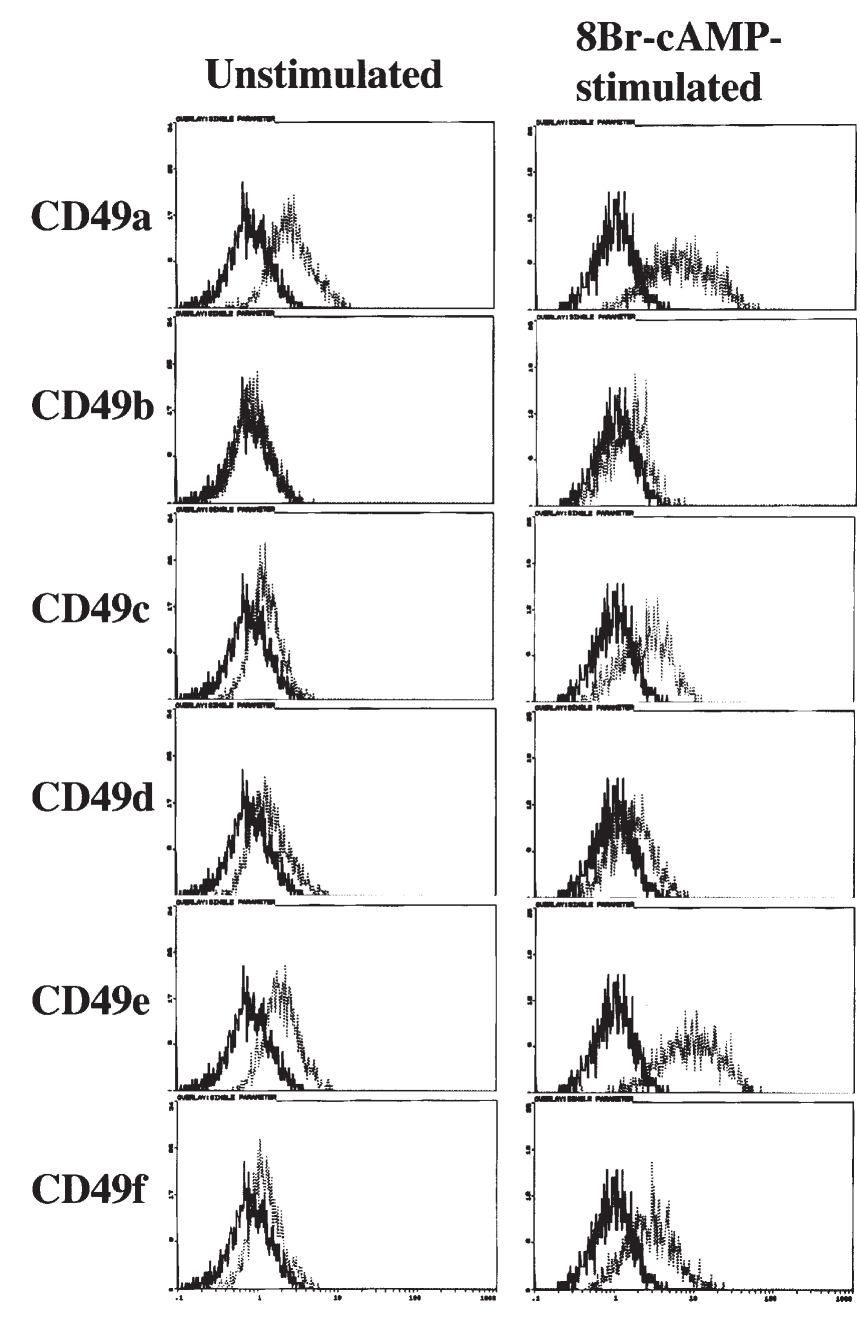

Figure 3. Expression profiles of integrin subunits, receptors for ECMcomponents, in normal human ESCs. Unstimulated ESCs and 8Br-cAMPstimulated ESCs were analyzed by semiquantitative flow cytometry. Unstimulated ESCs express low levels of CD49a, CD49c, CD49d, CD49e and CD49f, while 8Br-cAMP-stimulated ESCs express higher levels of these integrins on the cell surface. Marked increases are noted in the expression levels of CD49a, CD49c, CD49e and CD49f after 8Br-cAMP stimulation. The thick lines in all figures are negative controls while the thin lines show the integrin expressions.

individual ECM component-coated culture plates were observed. ESCs on fibronectin-coated dishes showed a large difference in their cell colony structures compared to ESCs cultured on other ECM component-coated dishes. Specifically, scattered ESCs proliferated on fibronectin-coated dishes while clusters of ESCs proliferated on type I collagen-, type IV collagen- and laminin-coated culture dishes (Fig. 4A and B). Although ESCs proliferated with bulges in the cell layer on type I collagen- and type IV collagen-coated dishes, the ESCs on laminin-coated dishes proliferated in a flat layer without any bulges (Fig. 4A and B). However, 8Br-cAMP-stimulated ESCs proliferated in a similar manner on the dishes coated with each of the four ECM components, with bulges in the cell layers and clustering of the cells (Fig. 4C and D).

Viability of ESCs cultured on individual ECM components. $8 \mathrm{Br}$-cAMP is not only a strong inducer of decidualization but also an inducer of activation-induced cell death. Many previous 


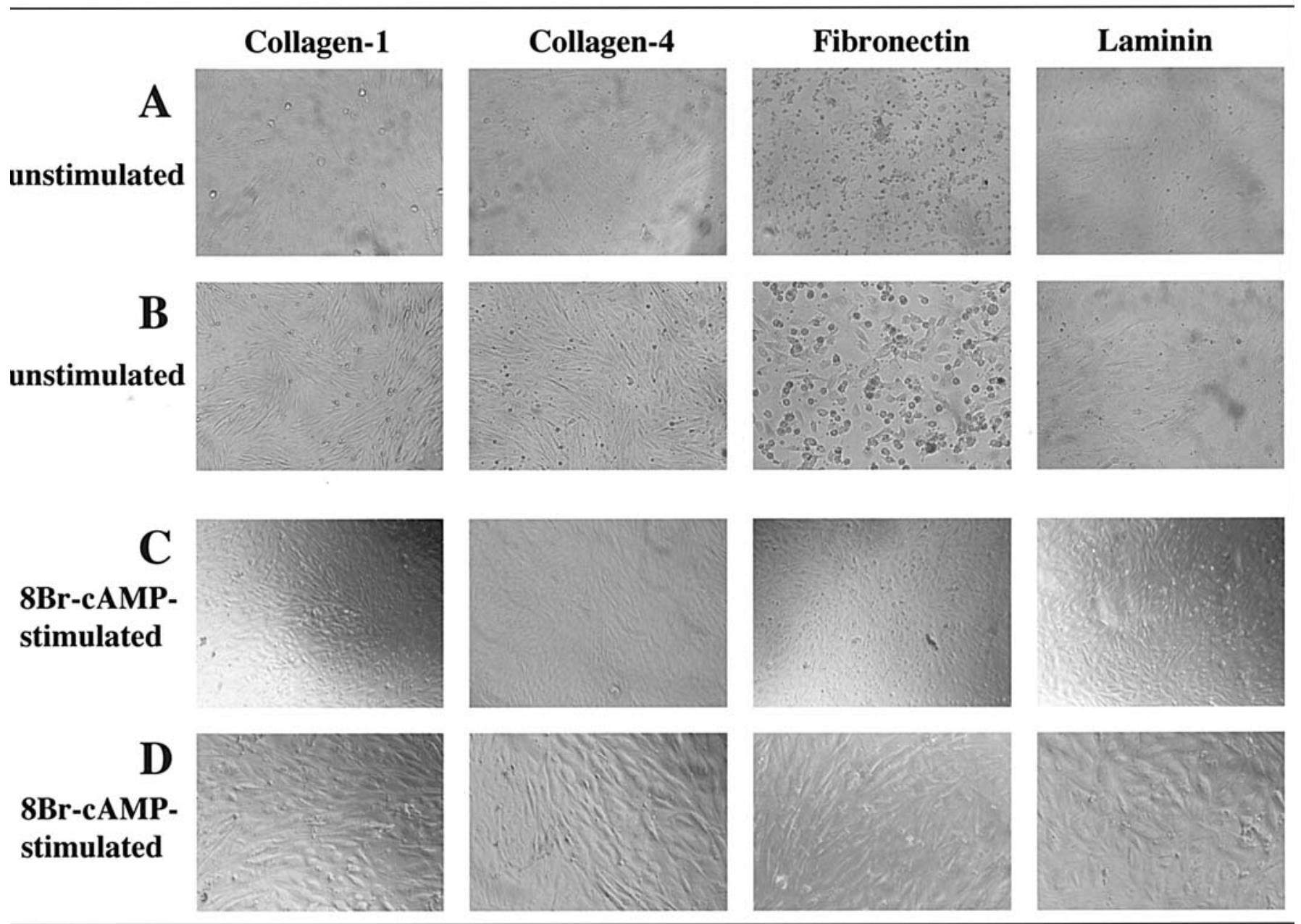

Figure 4. Microscopic findings for the cell sheet structures of normal human ESCs cultured on ECM component-coated dishes. The effects of ECM components on the cell sheet structures of cultured normal human ESCs were compared. (A and B) Unstimulated ESCs. (C and D) 8Br-cAMP-stimulated ESCs. Panel B is a magnified image of panel A. Panel D is a magnified image of panel C.
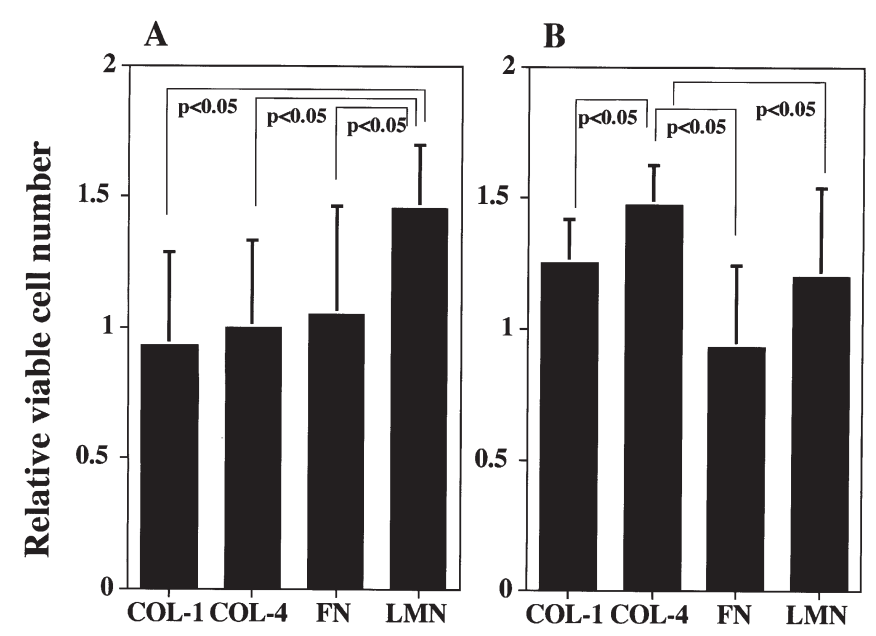

Figure 5. Effects of ECM components on the viabilities of $8 \mathrm{Br}$-cAMPstimulated ESCs. The viabilities of $8 \mathrm{Br}$-cAMP-stimulated ESCs cultured on ECM component-coated 96-well plates $(n=12)$ were assayed. (A) Relative numbers of viable $8 \mathrm{Br}$-cAMP-stimulating ESCs. (B) Relative numbers of viable $8 \mathrm{Br}$-cAMP-deprived ESCs after $8 \mathrm{Br}$-cAMP stimulation. reports have shown that integrin-mediated cell adhesion affects cell viability and proliferation (11-14). Furthermore, laminin and COL-4 are highly expressed in decidualized endometrial cells $(3,4)$. Therefore, we examined the effects of laminin and COL- 4 on the viabilities of $8 \mathrm{Br}$-cAMPstimulating ESCs and 8Br-cAMP-deprived ESCs. 8BrcAMP-stimulating ESCs cultured on laminin-coated dishes showed significantly higher viability than $8 \mathrm{Br}-\mathrm{cAMP}-$ stimulating ESCs cultured on dishes coated with the other three ECM components (Fig. 5A). However, regarding 8BrcAMP-stimulated ESCs subsequently cultured in 8Br-cAMPfree media, the cells cultured on COL-4-coated dishes showed the highest viability among the examined cells (Fig. 5B).

\section{Discussion}

The present study has demonstrated that specific adhesion between ECM components and ESCs can regulate the viability and proliferation mode of ESCs. We also showed that the productions of COL-4 and laminin by ESCs changed cyclically during the menstrual cycle, indicating that ovarian steroid hormones may regulate the expression levels of ECM components in ESCs. The production levels of COL-4 and 
laminin by decidualized ESCs were very high. Moreover, our experiments involving 8Br-cAMP-induced decidualization revealed that the viabilities of $8 \mathrm{Br}$-cAMP-stimulating ESCs and $8 \mathrm{Br}$-cAMP-deprived ESCs were differentially regulated by individual ECM components. Laminin was previously reported to inhibit the decidualization of human ESCs (5-6). Collectively, ovarian steroid hormones appear to regulate the proliferation, structure, differentiation and viability of ESCs by controlling their cyclic production of ECM components, similar to the autocrine/paracrine mechanisms utilized by the endocrine system.

Our immunohistochemical studies revealed that human ESCs, but not epithelial cells, were immunopositive for COL-4 and laminin. These findings suggest that, in the human endometrium, COL-4 and laminin are produced by stromal cells, but not epithelial cells, and are then transported to intercellular spaces and sub-epithelial basement membranelike structures. COL-4-positive and laminin-positive ESCs increased significantly during the LP and LS periods, while few postmenopausal stromal cells were positively stained for these two components. These results suggest that ovarian steroid hormones mainly regulate the productions of COL-4 and laminin by ESCs. Moreover, decidualized ESCs showed not only the highest positivities for COL-4 and laminin but also the strongest immunoreactivities for these components among all the tissues examined, suggesting that COL-4 and laminin may play their most important biological roles in decidualized stromal tissues.

Our flow cytometric analyses revealed that normal human ESCs expressed integrins, which act as receptors for ECM components, on their cell surface. In the cell-ECM component interaction experiments, ESCs proliferated with specific cell colony structures that varied depending on the ECM component coated on the culture dish. These results indicate that the cell surface integrins on normal ESCs act as specific functional receptors for ECM components. Notably, when ESCs were stimulated with $8 \mathrm{Br}$-cAMP, the cell surface integrin expression levels increased and it became difficult to identify morphological differences among the cell colony structures on the various ECM components. The increased integrin expression levels on the 8Br-cAMP-stimulated ESCs may have enhanced cell adhesion to the ECM components and thereby masked any differences in the cell colony structures identified by microscopy.

$8 \mathrm{Br}-\mathrm{cAMP}$ is a very strong decidulization inducer for ESCs, and subsequently induces activation-induced cell death. Therefore, we counted the numbers of viable $8 \mathrm{Br}$ cAMP-stimulating and 8Br-cAMP-deprived ESCs cultured on ECM component-coated dishes. 8Br-cAMP-stimulating ESCs exhibited higher viability on laminin-coated plates but significantly lower viability on collagen-coated plates. In contrast, $8 \mathrm{Br}$-cAMP deprivation reduced the viability of $8 \mathrm{Br}$ cAMP-stimulated ESCs on laminin-coated plates and significantly increased their viability on COL-4-coated plates. $8 \mathrm{Br}$-cAMP is a strong inducer of decidualization (15) and laminin was reported to inhibit the decidualization induced by 8Br-cAMP (6). Collectively, the differentiation and viability of ESCs in the LS endometrium with many decidualized cells may be autoregulated by laminin and COL-4 that are abundantly produced by ESCs in the decidua.
ESCs also express Fas antigen, a well-known death receptor, on their cell surface and the cell surface expression levels of Fas antigen are enhanced by $8 \mathrm{Br}$-cAMP-stimulation (16). Notably, Fas antigens on ESCs mediate cell survival signals but not apoptotic signals (16). Selam et al (17) reported that Fas ligand expression can be induced by cell adhesion to ECM components such as laminin and COL-4. Therefore, the increased productions of laminin and COL-4 in decidualized tissue and LP ESCs may regulate the Fas ligand expression in ESCs and the viability of ESCs by the Fas-Fas ligand system, and enable the maintenance of stable endometrial stromal tissue structures.

In summary, ECM components cyclically produced by ESCs during the menstrual cycle appear to stabilize endometrial tissue structures and regulate various functions of ESCs. The markedly increased levels of ECM components in decidualized tissue may play important roles in embryo implantation into the decidua. Abnormal expression levels of laminin and COL-4 in the endometrium may be a cause of infertility in patients.

\section{Acknowledgements}

This study was supported in part by a Grant-in-Aid for scientific research from the Ministry of Education, Science, Sports and Culture of Japan.

\section{References}

1. Bilalis DA, Klentzeris LD and Fleming S: Immunohistochemical localization of extracellular matrix proteins in luteal phase endometrium of fertile and infertile patients. Hum Reprod 11: 2713-2718, 1996.

2. Aplin JD, Charlton AK and Ayad S: An immunohistochemical study of human endometrial extracellular matrix during the menstrual cycle and first trimester of pregnancy. Cell Tissue Res 253: 231-240, 1988.

3. Wewer UM, Damjanov A, Weiss J, Liotta LA and Damjanov I: Mouse endometrial stromal cells produce basement-membrane components. Differentiation 32: 49-58, 1986.

4. Iwahashi M, Muragaki Y, Ooshima A and Nakano R: Decreased type IV collagen expression by human decidual tissues in spontaneous abortion. J Clin Endocrinol Metab 81: 2925-2929, 1996.

5. Brar AK, Frank GR, Richards RG, et al: Laminin decreases PRL and IGFBP-1 expression during in vitro decidualization of human endometrial stromal cells. J Cell Physiol 163: 30-37, 1995.

6. Mizuno K, Tanaka T, Umesaki $\mathrm{N}$ and Ogita S: Inhibition of cAMP-mediated decidualization in human endometrial stromal cells by IL-1ß and laminin. Horm Metab Res 31: 307-310, 1999.

7. Puistola U, Roennberg L, Martikainen H and TurpeenniemiHujanen T: The human embryo produces basement membrane collagen (type IV collagen)-degrading protease activity. Hum Reprod 4: 309-311, 1989.

8. Autio-Harmainen H, Sandberg M, Pihlajaniemi T and Vuorio E: Synthesis of laminin and type IV collagen by trophoblastic cells and fibroblastic stromal cells in the early human placenta. Lab Invest 64: 483-491, 1991.

9. Romagnano L and Babiarz B: The role of murine cell surface paractisyltransferase in trophoblast: laminin interactions in vitro. Dev Biol 141: 254-261, 1990.

10. Qin L, Wang YL, Bai SX, et al: Temporal and spatial expression of integrins and their extracellular matrix ligands at the maternal-fetal interface in the rhesus monkey during pregnancy. Biol Reprod 69: 563-571, 2003.

11. Park CC, Zhang H, Pallavicini M, et al: Beta 1 integrin inhibitory antibody induces apoptosis of breast cancer cells, inhibits growth, and distinguishes malignant from normal phenotype in three dimensional cultures and in vivo. Cancer Res 66: 1526-1535, 2006. 
12. Zhao X, Wang D, Zhao Z, et al: Caspase-3-dependent activation of calcium-independent phospholipase A2 enhances cell migration in non-apoptotic ovarian cancer cells. J Biol Chem 281: 29357-29368, 2006.

13. Edick MJ, Tesfay L, Lamb LE, et al: Inhibition of integrinmediated crosstalk with epidermal growth factor receptor/Erk or Src signaling pathways in autophagic prostate epithelial cells induces caspase-independent death. Mol Biol Cell 18: 2481-2490, 2007.

14. Edderkaoui M, Hong P, Lee JK, et al: Insulin-like growth factor-I receptor mediates the prosurvival effect of fibronectin. J Biol Chem 282: 26646-26655, 2007.
15. Tang B, Guller S and Gurpide E: Cyclic adenosine 3',5'monophosphate induces prolactin expression in stromal cells isolated from human proliferative endometrium. Endocrinology 133: 2197-2203, 1993.

16. Tanaka T and Umesaki N: Fas antigen (CD95) mediates cell survival signals to regulate functional cellular subpopulations in normal human endometrial stromal cells. Int J Mol Med 11: 757-762, 2003.

17. Selam B, Kayisli UA, Garcia-Velasco JA and Arici A: Extracellular matrix-dependent regulation of Fas ligand expression in human endometrial stromal cells. Biol Reprod 66: 1-5, 2002. 